\title{
Obtención de una bebida fermentada a partir de suero de queso utilizando células inmovilizadas de Kluyveromyces marxianus
}

\author{
Obtaining a fermented beverage from cheese whey by using \\ immobilized cells of Kluyveromyces marxianus
}

Rocío Cóndor G.' , Víctor Meza C. ${ }^{2}$ y Fanny Ludeña U. ${ }^{3}$

\section{RESUMEN}

El presente estudio tuvo como objetivo principal optimizar la tecnología de producción de una bebida iermentada de suero de queso, utilizando Kluyveromyces marxianus e inmovilización de células

En el biorreactor de lecho empacado, se obtuvo el mayor rendimiento $\left(11,46 \pm 0,81^{\circ} \mathrm{GL}\right) \mathrm{CC}$ un flujo de 140-150 mL. $h^{\circ}$. una biomasa inmovilizada de 10\% (v/v), una relación de columna de $i: 12$ (diámetro:longitud), tamaño de perlas de $3 \mathrm{~mm}$ de diámetro, adición de metabisulfito de sodio (100 $\mu \mathrm{g} . \mathrm{mL}^{\cdot 1}$ ) y células inmovilizadas con $2 \%(\mathrm{p} / \mathrm{v})$ de alginato.

Luego de optimizado todos los parámetros de fermentación en el biorreactor, la bebida alcohólica fue sometida a inactivación a $70^{\circ} \mathrm{C}$ por 5 minutos (tratamiento térmico) y clarificación con tierra de diatomeas $\left(1 \mathrm{~kg} \cdot \mathrm{m}^{-2}\right)$. Finalmente, la bebida alcohólica fue evaluada en almacenamiento por 6 meses, durante este periodo se le hicieron evaluaciones fisicoquímicas, microbiológicas y sensoriales. Se determinó por Escala Hedónica y Ranking que la bebida alcohólica de suero tiene aceptabilidad.

Palabras clave: Suero de queso, biorreactor, inmovilización, Kluyveromyces marxianus

\section{ABSTRACT}

This study has as its main objective the optimization of a technology for the production of a fermented beverage from cheese whey, using Kluyveromyces marxianus and cell immotilization.

In a bed packed reactor, the best yield was obtained $\left(11,46 \pm 0,81^{\circ} \mathrm{GL}\right.$ ) with a flux of $140-150 \mathrm{~mL} . \mathrm{h}^{-1}$, an immobilized biomass of $10 \%(\mathrm{v})$, a column rate of $1 / 12$, a size of pearl of 3 mm of diameter, an addition of sodium metabisulphide $\left(100 \mu \mathrm{g} \cdot \mathrm{mL}^{-1}\right)$ and immobilized cells with $2 \%$ (w/v) of alginate.

After the fermentation parameters were optimized in the bioreactor, the alcoholic beverage was inactivated at $70^{\circ} \mathrm{C}$ for 5 minutes (thermal treatment) and clarified with "tierra de diatomeas" $\left(1 \mathrm{~kg} \cdot \mathrm{m}^{-2}\right)$. Finally, the alcoholic beverage was evaluated in storage for 6 months, during this period physicochemical, microbiological and sensorial tests were made. Hedonic Scale and Ranking tests showed that the alcoholic beverage had acceptability.

Key words: Cheese whey, bioreactor, immobilization, Kluyveromyces maxianus

\section{INTRODUCCIÓN}

En el Perú, actualmente, en el campo de las industrias prociuctoras de queso, se tiene un proulema latente sin resolver, el cual se genera debido a la gran producción de suero

\footnotetext{
1 Investigadara del Laboratario de Microbiologia "Marino Tabusso". Dopartamento de Biologia. UNALM

2 Profesor Auxiliar. Departamento de Biologia. UNALM

3 Prolesora Asociada Depanamento de Tecnología de Aimentos y Planta Piloto CELEche, UNALM
}

compuesto que se desecha a través del alcantarillado municipal generando problemas de contaminación ambiental. Para tener una idea de la cantidad de suero que se produce sólo en la Universidad Nacional Agraria La Molina (UNALM), se sabe que la Planta Piloto de Leche destina entre 270 a 750 litros de leche para la producción de quesos, por lo que se obtienen entre 235.17 a 653,25 litros de suero, diariamente. 
Alrededor de 45 kilos de leche cruda darán 4,5 kilos de Demanda Bioquímica de OxígenoDBO (Braile, 1993). Con el fin de controlar y reducir las descargas de desechos líquidos de las industrias productoras de queso, se podría mejorar el proceso de producción de la planta, tratar los desechos líquidos o aprovecharlos.

En el presente trabajo, se buscó aprovechar el suero de quesería de la Planta Piloto de Leche de la UNALM, utilizando la tecnología de inmovilización de células para la producción de una bebida fermentada. Se establecen también los parámetros necesarios para que se pueda optimizar su producción. Resaltando la importancia de la Biotecnología en la optimización de procesos para el mejoramiento del aprovechamiento del suero.

En la actualidad, el consumo de bebidas lácteas a partir de suero está muy difundida por su valor nutritivo y menor costo (Kriel, 1980; Sabaa-Srur el al., 1995). Industrialmente el suero sirve como ingrediente en la elaboración del kefir (Garibayel al., 1993), koumiss (Garibay et al., 1993) y bebidas lácteas con frutas (Fresnel y Moore, 1978). Otra línea de producción creciente son las bebidas lácteas fermentadas con bacterias o mezclas de éstas con levaduras, las cuales generalmente se mezclan con jugos u hortalizas u otros saborizantes (Garibay et al., 1993).

\section{MATERIAL Y MÉTODOS}

El presente estudio se realizó en el Laboratorio de Microbiología "Marino Tabusso" de la Facultad de Ciencias y en la Planta Piloto de Leche de la UNALM. Se trabajó con suero de queso fresco, Kluyveromyces marxianus (Kluyveromyces fragilis NRRL Y-2415) y alginato de sodio (SIGMA).

\section{INMOVILIZACIÓN DE Kluyveromyces marxianus}

La levadura fue repicada de un tubo que contenía la cepa madre a un medio de cultivo en placa; se incubó a $30^{\circ} \mathrm{C}$ durante 5 días. Después se tomaron 20 asadas y se llevó a $200 \mathrm{~mL}$ de suero acondicionado ( $\mathrm{pH} 4,5$ y desproteinizado), contenidos en un erlenmeyer de un litro. Se incubó a $30^{\circ} \mathrm{C}$ durante 10 horas. Para la recuperación de las levaduras, el cultivo se centrifugó a 5000 rpm durante 10 minutos: las células sedimentadas fueron suspendidas y lavadas dos veces con una solución de peptona al $0,85 \%$ y se almacenaron a $4{ }^{\circ} \mathrm{C}$ hasta su utilización.

Para el proceso de inmovilización, se autoclavó $2,0 \mathrm{~g}$ de alginato de sodio con 30 $\mathrm{mL}$ de agua destilada; a esta mezcla se le agregó una suspensión de levaduras (se evaluaron diferentes concentraciones: 10, 20, $30 \% \mathrm{v} / \mathrm{v}$ ) y finalmente, se completó con la cantidad de agua destilada necesaria para llegar a $100 \mathrm{~mL}$ (concentraciones final: 10,20 ó 30\% de biomasa). La mezcla se dejó caer sobre una solución de cloruro de calcio 0,1M y se dejó polimerizar durante 1 hora. Posteriormente se trasladó las perlas formadas a una solución de cloruro de calcio $0,05 \mathrm{M}$ y se refrigero 24 horas. Finalmente se trasladó a una solución de cloruro de calcio 0,025M donde se mantuvieron hasta su uso en refrigeración (Marwaha et al., 1988). Antes de ser utilizadas, fueron lavadas con una solución salina para remover los iones de calcio que estuvieran en exceso (Chibata et al., 1987; Marwaha et al., 1988; Mc Ghee et al., 1982). Para conocer el número de células en cada perla. se dejó gotear $1 \mathrm{~mL}$ de la mezcla sobre agua. Luego se hizo el conteo de las células con la cámara de Neubauer, expresando los resultados en número de células. $\mathrm{mL}^{-1}$ de gel.

DETERMINACIÓN DEL EFECTO DE METABISULFITO DE SODIO SOBRE LA CINÉTICA DE FERMENTACIÓN CON DIFERENTES CONCENTRACIONES DE LEVADURA

Se evaluaron tres concentraciones de levadura: 10, 20 y $30 \%(\mathrm{v} / \mathrm{v})$; en sistemas $\sin$ metabisulfito y con $100 \mathrm{mg} . \mathrm{mL}^{-1}$ de metabisulfito. Se trabajó con $50 \mu \mathrm{L}$ de sustrato $\left(21^{\circ}\right.$ Brix) y 100 perlas con las diferentes con- 
centraciones de células. Se incubó a $30{ }^{\circ} \mathrm{C}$ por 7 días (Fresnel y Moore, 1978; Gawel y Kosikowski, 1978; Yang et al., 1979), evaluándose la cinética de fermentación con la lectura de grados Brix y pH (Núñez y Lema, 1987).

Determinación del flujo Óptimo en el BIORREACTOR DE LECHO EMPACADO

En una relación de columna diámetro:longitud de $1: 12(2,2: 26,4 \mathrm{~cm})$, se empacó 70\% de su capacidad (3000 perlas): el contenido de biomasa de las perías fue de $10 \%$ $(\mathrm{v} / \mathrm{v})$, lo que represcintó un rango de $5.3 \times 10^{*}$ a $1,2 \times 10^{9}$ células. $\mathrm{mL}^{-1}$ de gel. El suero

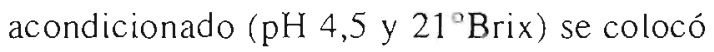
en el depósito superior del lecho. Se evaluaron 4 rangos de flujo: 50-60; 90-100; 140-150 y 240-250 mL.h ${ }^{-1}$ (Marwaha et al., 1988). Se trabajó todo el proceso de fententación a $30^{\circ} \mathrm{C}$, evaluándose diariamente los grados Brix y el pH (Ough, 1996). Se determinó el flujo óptimo, mediante el rendimiento nudido en grados a)cohólicos al final de la fermentación.

Determinación de la Biomasa óptima para EL PROCESO FERMENTATIVO

Determinado el flujo óptimo, se probu tres concentraciones de biomasa: 10,20 y $30 \%(\mathrm{v} / \mathrm{v})$, conteniendo un rango de $5.3 \times 10^{\mathrm{s}}$ a $1.2 \times 10^{4}$; $1,1 \times 10^{9}$ a $1,3 \times 10^{9}$ y $2,0 \times 10^{9}$ a $3,0 \times 10^{\prime \prime}$ células. $\mathrm{mL}^{-1}$ de gel, respectivamente. Se empacó perlas hasta un $70 \%$ de la capacidad de la columna (1:12). Se evaluó diariamente la concentración de azúcares ( ${ }^{\circ} \mathrm{Brix}$ ) y el pH. El grado alcohólico se evaluó después de 7 días de fermentación.

DETERMINACIÓN DE LA DIMENSIÓN ÓF TIMA DE LA COLUMNA

Después de determinar el flujo y concentración de biomasa óptimos, se evaluó tres relaciones de columna 1:12; 1:15 y $1: 17$ (diámetro: longitud) que correspondían con las dimensiones de 2,2:26,4 cm;2,2:33,0 cm y 2,2:37,4 cm, respectivamente. Se empacó perlas hasta un $70 \%$ de la capacidad de la dimensión de la columna. Se evaluó diariamente la fermentación con el control del grado Brix, pH y —después de 7 días-el grado alcohólico.

\section{INACTIVACIÓN Y CLARIFICACIÓN DE LA BEBIDA FERMENTADA}

La inactivación de la bebida fermentada se hizo a $70^{\circ} \mathrm{C}$ durante 5 minutos (Cóndor et al., 2000). Para realizar la clarificación, se empleó tierra de diatomeas en una cantidad de $1 \mathrm{~kg} \cdot \mathrm{m}^{-2}$ (Molina, 1992). Se realizó la desinfección y clarificación por procedimientos descritos anteriormente (Cóndor et al., 2000). Finalmente, se agregó $50 \mu \mathrm{g} . \mathrm{mL}^{-1} \mathrm{de}$ metabisulfito de sodio a la bebida, para su conservación.

\section{Caracterización fisicoquimica y microbIológica} DE LA BEBIDA FERMENTADA DE SUERO DE QUESO

Evaluaciones fisicoquímicas: humedad (Pearson, 1976); cenizas (Amerine y Ough, 1976); pH (Amerine y Ough, 1976); acidez total, volátil y fija (Amerine y Ough, 1976); sólidos solubles totales (Amerine y Ough, 1976); azúcares totales y reductores (AOAC, 1995); sulfuroso total y libre (Amerine y Ough, 1976); grado alcohólico (Amerine y Ough, 1976); proteínas (AOAC, 1990).

Se realizaron las siguientes evaluaciones microbiológicas: recuento de mesófilos aerobios viables; numeración de coliformes totales y recuento de mohos y levaduras. Se tomó como referencia la Norma Técnica Peruana 202.083 (1988).

\section{Evaluación SENSORIAL dE LA BEBIDA FERMENTADA}

La caracterización organoléptica se efectuó tomando en consideración la Norma ISO $4121-1987$

La evaluación sensorial se llevó a cabo con 24 panelistas semientiunados (Laboratorio de Análisis Sensorial-Programa de Nutrición Humana. UNALM). Se determinó la aceptación del producto con dos métodos: Escala Hedónica no estructurada (Amerine, 1965; Pedrero y Pangborn, 1989) y cl método de 
Ranking (Pedrero y Pangborn, 1989). Como referencia se utilizó tres vinos de características similares. Los resultados fueron evaluados estadísticamente (Pedrero y Pangborn, 1989).

\section{Evaluación organoléptica durante el ALMACENAMIENTO}

La bebida alcohólica envasada en botellas de $400 \mathrm{~mL}$ de capacidad fue almacenada durante cinco meses bajo dos condiciones: temperatura ambiente (botella transparente y oscura) y en refrigeración (botella transparente). Durante este período se evaluaron la acidez total y el $\mathrm{pH}$.

\section{RESULTADOS Y DISCUSIÓN}

EfEcto del METABisulfito de SODIo SOgRe La CINÉTICA DE FERMENTACIÓN CON DIFERENTES CONCENTRACIONES DE LEVADURA

Se determinó que la cinética de fermentación con células inmovilizadas (diferentes concentraciones de biomasá: 10,
20 y $30 \%$ ), en sistemas sin y con adición de metabisulfito de sodio (100 $\left.\mu \mathrm{g} \cdot \mathrm{mL}^{-i}\right)$ es diferente. Se puede observar en la Fig. 1 que en sistemas sin adición de metabisulfito, existe una relación directa entre la biomasa inmovilizada y la cantidad de azúcares totales ( ${ }^{\circ}$ Brix) que se consume. La mayor disminución de azúcares, se obtiene con $30 \%$ (v/v) de levaduras inmovilizadas. Sin embargo, en sistemas con metabisulfito se produce una relación inversa; se obtuvo una mayor disminución de azúcares con $10 \%(v / v)$ de levaciuras.

Los resultados demuestran que $K$. marxiauns se ve afectada por la adición del mciabisulfito, y si a esto se suma la inmovilización de células, el efecto es mayor. debido a que la inmovilización limita la difusión de nutrientes. Además, las perlas están en un cultivo en lote sin ningún tipo de agitación, por eso tienen una menor transferencia de masá, lo cual se acentúa con el aumento de la concentración de biomasa inmovilizada. Por otro lado, se sabe que existe una relación física

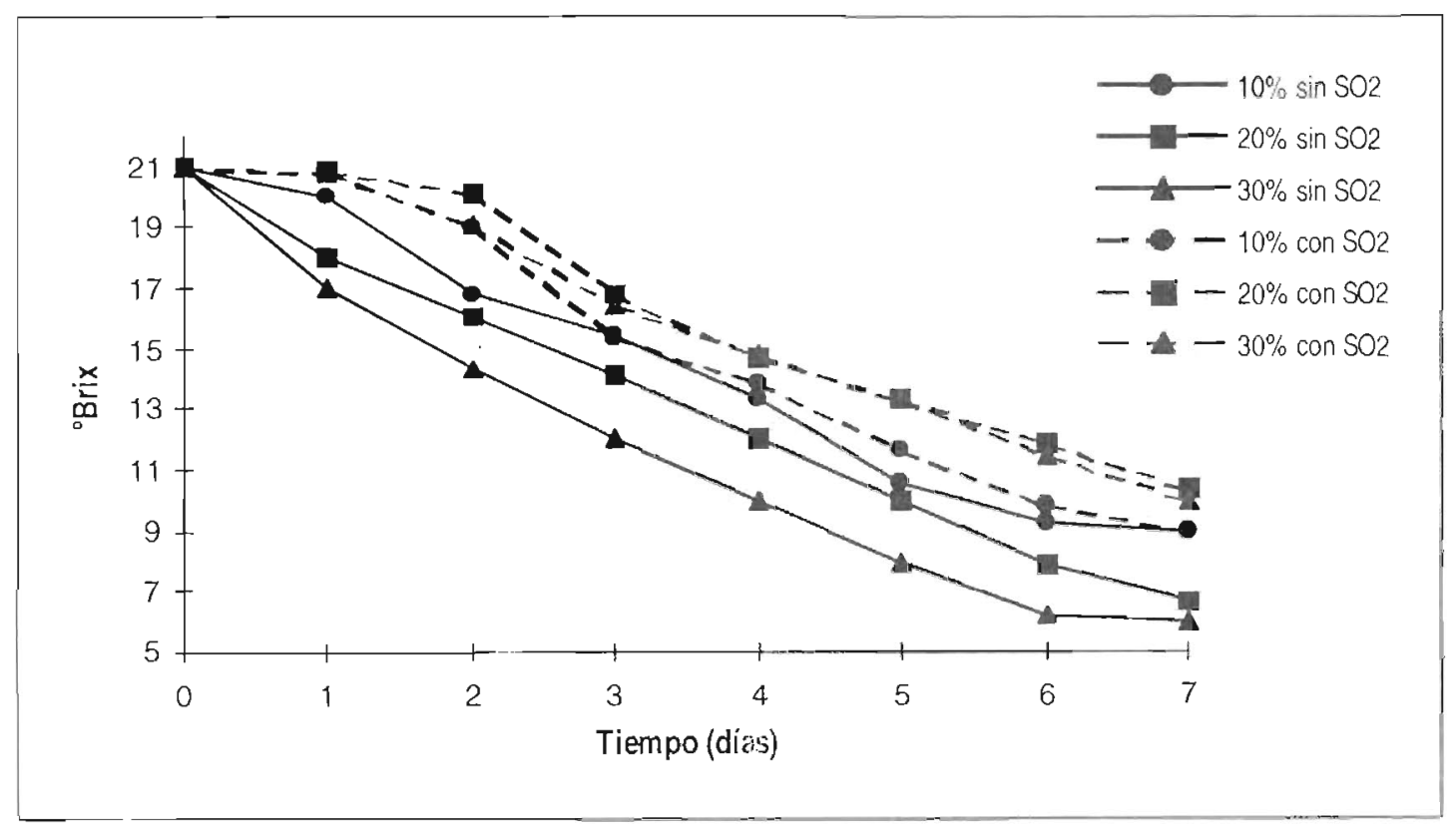

Fig. 1 Variación de la concentración de azúcar ( ${ }^{\circ}$ Brix) durante la fermentación con diferentes concentraciones de levadura, en sistemas sin y con adición de metabisulfito de sodio 
entre las perlas, las cuales se ven modificadas en superficie e interfase por la acumulación de $\mathrm{CO}_{2}$ (Mc Ghee et al., 1988).

\section{FLUJO ÓPTIMO PARA OBTENCIÓN DE LA BEBIDA FER- MENTADA}

Se determinó que el mayor rendimiento $\left(11,46 \pm 0,81{ }^{\circ} \mathrm{GL}\right)$ para la producción de la bebida fermentada de suero se obtiene con un flujo de 140-150 mL.h-1 (ver Fig. 2). De manera similar, se ha reportado un flujo de 142 $\mathrm{mL} \cdot \mathrm{h}^{-1}$ como óptimo para la producción de alcohol a partir de suero en un biorreactor de lecho empacado (Marwaha et al., 1988). El valor obtenido puede deberse a que en estas condiciones la levadura $K$. marxianus logra una mejor transferencia de masa. transformando los azúcares presentes en el medio. Además, debe generarse la suficiente turbulencia como para permitir la remoción de catabolitos que pueden inhibir la actividad de las levaduras, en biorreactores con células inmovilizadas (Dale ét al., 1994; Núñez y Lema, 1987; Parascandola et al., 1997). Al ser comparados los rendimientos entre células inmovilizadas y libres de $K$. marxianus, se observa un mayor rendimiento para las primeras, con valores de grado alcohólico de $11,46 \pm 0,8\rfloor$ y $9,83 \pm 0,55^{\circ} \mathrm{GL}$, respectivamente. Se ha reportado que la mayor concentración de etanol se obtiene con células inmovilizadas que con células libres en fermentaciones con $K$ marrianus IMB3 y sacarosa (Ferguson et al. 1998).

\section{Biomasa óptima para la obTención de la beBida ALCOHÓLICA}

Se determinó que el mayor rendimiento $\left(11,46 \pm 0,81^{\circ} \mathrm{GL}\right)$ para la producción de la bebida fermentada de suero se obtiene con una biomasa de $10 \%(\mathrm{v} / \mathrm{v})$ (ver Fig. 3), con respecto a las concentraciones de 20 y $30 \%$ respectivamente. Esto se clebe a que las altas concentraciones de levaduras ocasionan una tasa de consumo de sustrato superior a la tasa de transferencia de producto al exterior de la perla, lo cual puede inhibir a las levaduras inmovilizadas. Se senala que una mayor concentración de levaduras inmovilizadas por unidad de gel en las perlas de alginato

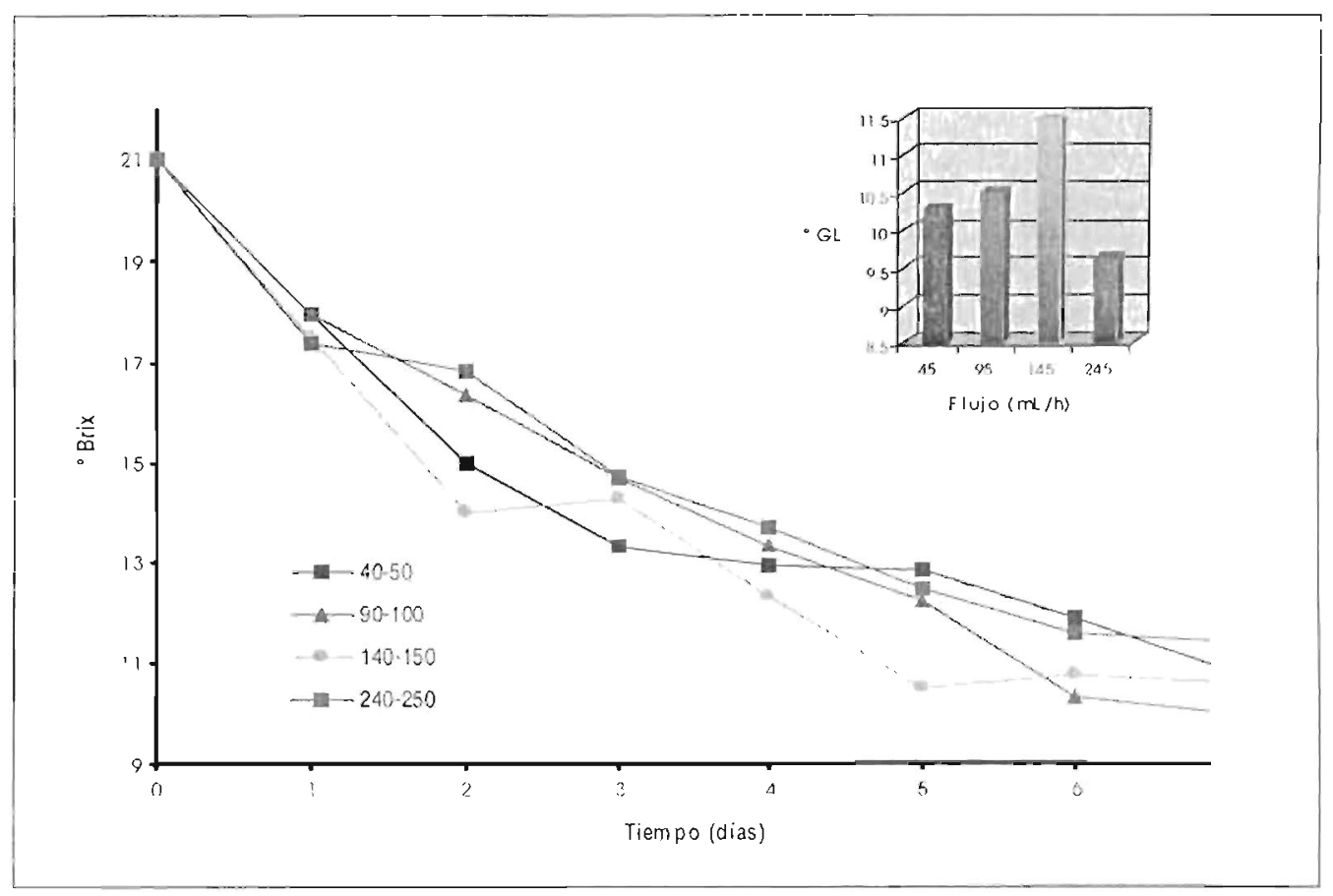

Fig. 2 Variación de la concentración de azúcar ( ${ }^{\circ}$ Brix) durante la fermentación con diferentes flujos de inmovilización 
disminuye el coeficiente de difusión del sustrato y productos (Divies et al., 1994). Además, el sustrato, para ser utilizado por las células inmovilizadas y empacadas en una columna, tiene que pasar por varias barreras; primero atraviesa la interfase perla y medio líquido, luego habrá limitaciones difusionales dentro de la perla y finalmente el sustrato atravesará la pared celular (Wiseman, 1991).

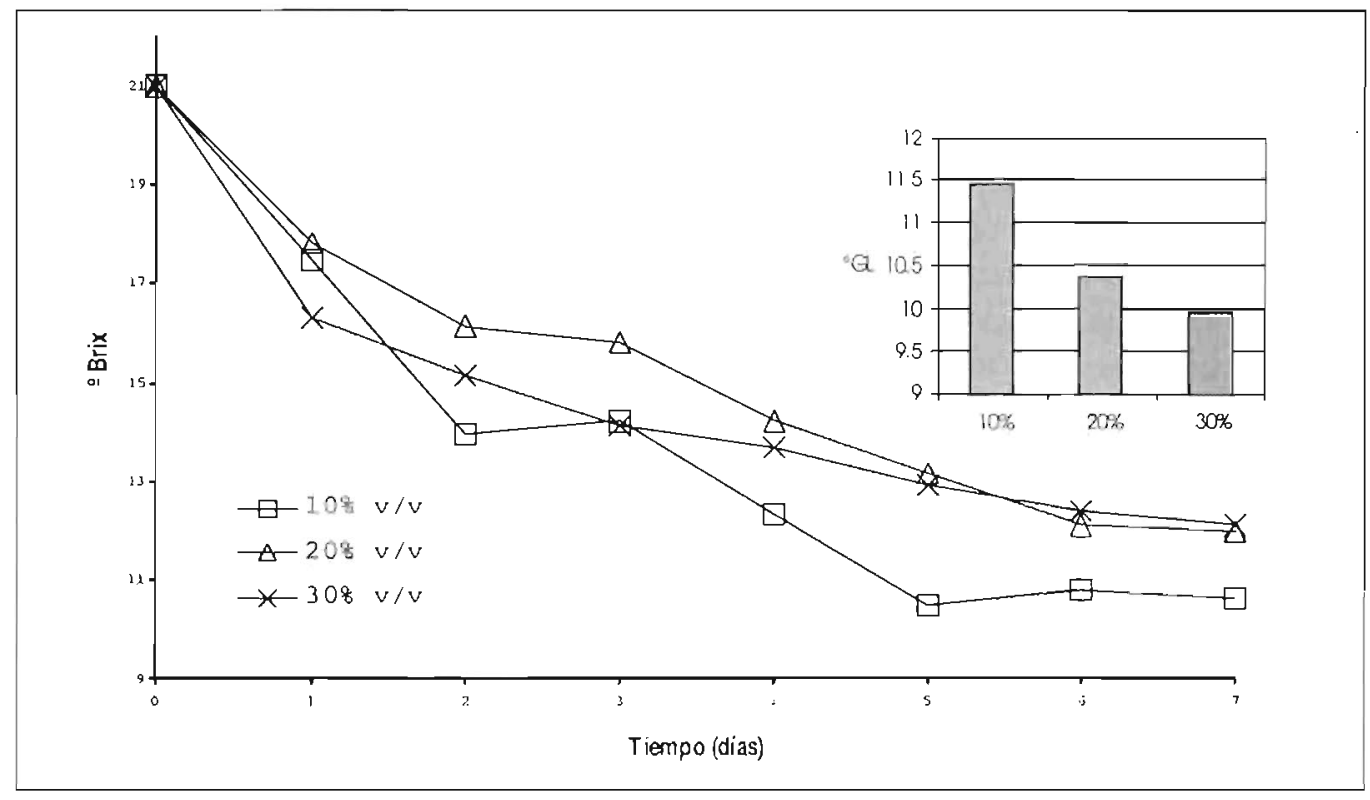

Fig. 3 Variación de la concentración de azúcar $\left({ }^{\circ} B r i x\right)$ durante la fermentación con diferentes concentraciones de levadura $(\% \mathrm{v} / \mathrm{v})$

Tabla 1. Evaluación fisicoquímica de la bebida fermentada

\begin{tabular}{|c|c|}
\hline ANÁLISIS & BEQDA ALCOHOLICA \\
\hline Humedad (\%) & $92,87 \pm 0,84$ \\
\hline Cenizas $\left(g . l^{-1}\right)$ & $4,46 \pm 0,58$ \\
\hline $\mathrm{pH}$ & $3,8 \pm 0,90$ \\
\hline $\begin{array}{l}\text { Acidez lolal, expresada en } \\
\text { g.t'ácido sulhirico }\end{array}$ & $3.25 \pm 0.05$ \\
\hline $\begin{array}{l}\text { Acidez volátil, expresada en } \\
\text { g.l" ácido acético }\end{array}$ & $0,48 \pm 0,33$ \\
\hline Acidez fija(g. $\left.\left.\right|^{1} \mathrm{H} 2 \mathrm{SO} 4\right)$ & $2,62 \pm 0,35$ \\
\hline Sólidos solubles ( ${ }^{\circ} \mathrm{Brix}$ ) & $10,77 \pm 0,87$ \\
\hline Azicar total & $5,50 \pm 0,10$ \\
\hline Azucar reductor & $5,14 \pm 1,17$ \\
\hline Sulfuroso total (mg.mL') & $64,00 \pm 1,20$ \\
\hline Suffuroso libre (mg.mL $\left.\mathrm{mL}^{-1}\right)$ & $16,00 \pm 2,40$ \\
\hline $\begin{array}{l}\text { Sulfuroso combinado } \\
\left.\text { (mg. } \mathrm{mL}^{-1}\right)\end{array}$ & $48,00 \pm 1,40$ \\
\hline Grado alcohólico ( $\left.{ }^{\circ} \mathrm{GL}\right)$ & $11,47 \pm 0,81$ \\
\hline Proteina $(\%)$ & 0,10 \\
\hline
\end{tabular}


El empleo de altas concentraciones de células entrampadas en alginato acorta el tiempo requerido para la producción del máximo rendimiento de alcohol; sin embargo, el porcentaje de células que sobreviven decrece con el aumento de la concentración inicial de células (Yousef et al., 1989). Las levaduras inmovilizadas se pueden ver afectadas en su rendimiento por efectos de la gran producción de $\mathrm{CO}_{2}$ por unidad de perla. La producción de $\mathrm{CO}_{2}$ es 2,5 veces mayor en células inmovilizadas en alginato que en células libres a 5 y $10 \%$ de etanol (Ciesarova et al., 1998). Con respecto a la adición de metabisulfito y diferentes concentraciones de levadura inmovilizada, se obtiene un perfil muy parecido de disminución de azúcares al obtenido en cultivos en lote. Una mayor disminución de azúcares totales con la menor concentración de biomasa, $10 \%(\mathrm{v} / \mathrm{v})$.

RELACIÓN ÓPTIMA dE LA COLUMNA PARA LA OBTENGIÓN DE LA BEBIDA ALCOHÓLICA

Se determinó que el mayor rendimiento de grados alcohólicos $\left(11,46 \pm 0,81^{\circ} \mathrm{GL}\right)$ en la producción de la bebida fermentada se obtiene con una relación cle columna de 1:12 (ver Fig. 4). Esto podría deberse a que se generan dimensiones intermedias que permiten una buena transferencia de masa y una eficiente liberación de $\mathrm{CO}_{2}$. Experimentos realizados con células inmovilizadas en alginato y en lecho empacado determinaron que las dimensiones del biorreactor afectan la productividad y eficiencia de la fermentación debido al $\mathrm{CO}_{2}$ y efectos de la transferencia de masa (Buzas et al., 1990; Yadav et al., 1996).

Por otro lado, la relación de columna 1:12 permite dimensiones que hacen que se mantengan condiciones equivalentes en cada parte de la columna, de tál manera que las perlas que están ubicadas en la base de la columna presentan igual conversión de azúcares que las perlas ubicadas en la parte superior. Probablemente. las otras relaciones de columna (1:15 y 1:17) no presentan condiciones similares a lo largo de la columna, pudiéndose afectar las perlas que están en la base de la columna, porque soportan una mayor presión física.

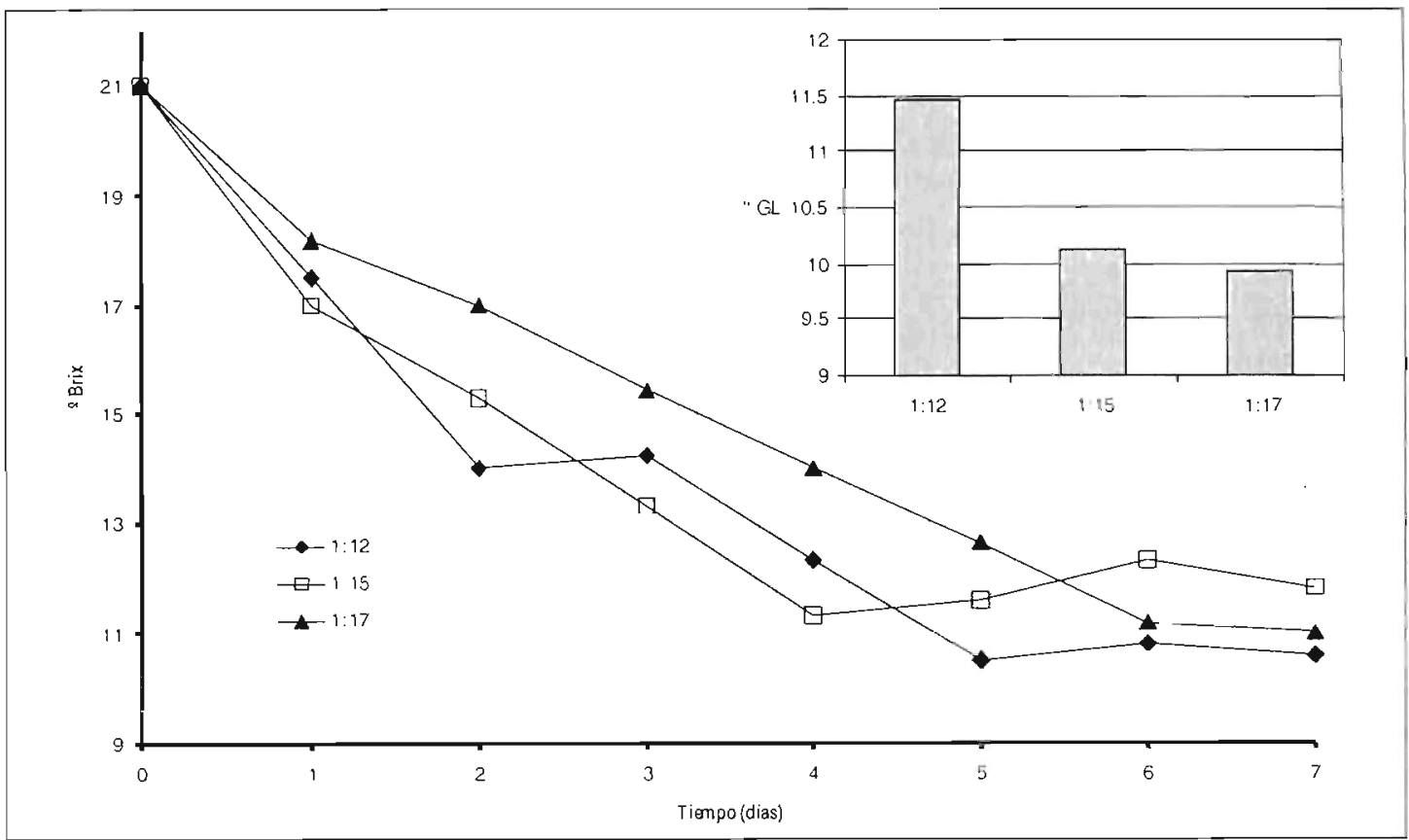

Fig 4 Variación de la concentración de azúcar ( ${ }^{\circ}$ Brix) durante la fermentación con diferentes dimensiones de la columna 
Caracterización fisicoquimica de la begida ALCOHÓLICA DE SUERO

Los resultados se presentan en el Tabla 1. Los valores determinados están dentro de los rangos permitidos para bebidas alcohólicas similares (NTP, 1995; Ough, 1996; Peynaud, 1989; Saaba-Srur et al., 1995).

\section{Caracterización microbiológica}

Después del almacenamiento de la bebida, se ha obtenido un recuento menor a 10 de mesófilos aerobios viables (UFC. $\mathrm{mL}^{-1}$ ); un recuento menor a 10 de mohos y levaduras (UFC. $\mathrm{mL}^{-1}$ ) y una numeración menor a 3,0 de coliformes totales (NMP.mL-1). Esto indica que la bebida es microbiológicamente aceptable y que los métodos de inactivación y estabilización efectuados son los adecuados.

\section{Comportamiento en almacenamiento}

Después de terminada la fermentación, se eliminaron las levaduras mediante clarificación con tierra de diatomeas $\left(1 \mathrm{~kg} \cdot \mathrm{m}^{-2}\right)$. Luego se evaluó el comportamiento en almacenamiento a temperatura ambiente y refrigeración, con $\mathrm{pH}$ iniciales de 3,4 y acidez total de de $3,6 \mathrm{~g} / \mathrm{l}$ $\mathrm{H}_{2} \mathrm{SO}_{4}$.

Se tuvo en el primer mes un descenso en acidez total y un aumento del $\mathrm{pH}$. Esta pérdida de acidez total inicial se puede deber a la precipitación 'de sales, proteínas y ciertos compuestos que son insolubles a bajas temperaturas, y pueden modificar las características microbiológicas y organolépticas de la bebida. Además, a bajas temperaturas se facilita la solubilidad de los gases disueltos (Peynaud, 1989).

A partir del segundo mes, hasta los cinco meses que duró la evaluación, se observó un perfil variable del $\mathrm{pH}$ y acidez total. En general a temperatura ambiente $\left(22^{\circ} \mathrm{C}\right)$ y en refrigeracion $\left(4^{\circ} \mathrm{C}\right)$ las características evaluadas de la bebida fermentada presentaron un comportamiento similar. Se obtuvieron valores finales a temperatura ambiente $y$ refrigeración, $\mathrm{pH} \quad 3,7$ y 3,9 y acidez total de 3,2 y $3,3 \mathrm{~g} / \mathrm{H} \mathrm{H}_{2} \mathrm{SO}_{4}$, respectivamente.

Luego, se evaluó la bebida fermentada en botellas transparentes y oscuras, y se determinó que éstas últimas mantienen sus características organolépticas.

\section{EVALUACION SENSORIAL DE LA BEgIDA FERMENTADA}

La caracterización organoléptica realizada en un Laboratorio Certificado indica que la bebida alcohólica de suero tiene un olor y sabor característicos ue un vino semiseco.

La evaluación de aceptación realizada con la prueba de Ranking, concluyó que las tres muestras evaluadas comparadas con el control (bebida alcohólica de suero) son inferiores. Con Escala Hedónica, se evaluó el atributo olor, color y sabor, y se obtuvo $77 \pm 2,83 ; 59 \pm$ 14,85 y $68,5 \pm 12,02 \%$ de aceptación, respectivamente, lo que indica solo discrepancia con el atributo color y sabor.

\section{CONCLUSIONES}

1. En un sistenua sin mutabisulfito de sodio, lá concentración óptima de células inmovilizadas es de $30 \%$ (v/v), y para un sistema con metabisulfito de sodio la concentración óptima es de $10 \%(\mathrm{v} / \mathrm{v})$.

2. El mayor rendimiento $\left(11,46 \pm 0,81^{\circ} \mathrm{GL}\right)$ en un biorreactor de lecho empacado se obtiene con las siguientes condiciones; un flujo de 140-150 mL.h. ${ }^{-1}$, una biomasa inmovilizada de $10 \%(\mathrm{v} / \mathrm{v})$, una relación de columna (diámetro:longitud) de $1: 12$ tamaño de perlas de $3 \mathrm{~mm}$, adición de metabisulfito de sodio ( $\left.100 \mathrm{mg} \cdot \mathrm{mL}^{-1}\right)$ y células inmovilizadas con $2 \%(\mathrm{p} / \mathrm{v})$ de alginato.

3. Optimizados los parámetros de producción de la bebida fermentada en un biorreactor de lecho empacado, se obiuvo una bebida 
con: $11,47 \pm 0,81^{\circ} \mathrm{GL} ; 0,48 \pm 0.33 \mathrm{~g} \cdot 1^{-1} \mathrm{de}$ acidez, expresado en ácido acético; $64 \pm$ $1,2 \mathrm{mg} \cdot \mathrm{mL}^{-1}$ de sulfuroso total; $0,46 \%$ de cenizas y $0,1 \%$ de proteínas en 7 días de ferinentación.

4. La calidad microbiológica de la bebida fermentada es aceptable.

5. Las botellas oscuras permitieron mantener las características organolépticas de la bebida fermentada.

6. La evaluación sensorial de la bebida alcohólica, tanto por Escala Hedónica como por Ranking, demuestra que la bebida tiene aceptación.

\section{LITERATURA CITADA}

Amerine, M. 1965. A series of munographs. Principles of sensory evaluation of Fuod. Academic Press S. A. USA. pp. 306-374.

Amerine, M. y C. OUGH. 1976. Análisis de vinos y mostos. Acribia $\mathrm{S}$. A. Zaragoza, $158 \mathrm{pP}$.

AOAC 28.1.14-1995. Vinos. Delerminación de azúcares en vinos.

AOAC 920.105-1990. I Jeterminación de proteínas. Método de Khejdal.

Braile, D.M. 1993. Manual de tratamiento de aguas Residuarias Industriáis. CETESB. Brazil, pp. 235-267.

Buzas, Z.; K. Dallman; L. Boross; B. Szajani \& G. Horvath. 1990. Factors influencing the operation of the vertical bioreactor segmented with perforaled plates. Acta Biochim. Biophys. Hung. 25( $1-2): 9-16$.
Ciesarova, Z.; Z. Domey; D. Smogrovicova; J. Patkova \& E. Sturdik. 1998. Comparison of ethanol tolerance of free and immobilized Saccharomyces uvarum yeasts. Folia Microbiol. (Praha) 43(1):55-58.

Chibata, I.; T. Tosa \& T. Sato. 1986. Methods of cell Immobilization. En "Manual of Industrial Microbiology and Biotechnology" (A. L. Demain \& N. A. Solomon, Eds.) American Society for Microbiology. Washington, DC, pp. 217-247.

Cóndor, R; V. Meza y F. Ludeña. 2000. Recuperación de suero de quesería para la producción de una bebida alcohólica en un sistema en lote. En prensa.

Dale, A; A. Eager \& M. Okos. 1994. Osmotic inhibition of free and immobilized $K$. marxianus: Anaerobic growth and ethanol productivity in whey permeate concentrate. Process Biochem, 29: $535-544$

Divies, C.; R. Cachon; J. Cavin \& H. Prescot. 1994. Theme 4: Immobilized Cell Technology in wine production. Critical Rev. in Biotech. 145(2): 135-153.

Ferguson, P.; H. Mulholland; N. Barrón; D. Brady \& A. Mc Hale. 1998. Sucrose-supplemented distilery wash as a medium for production of ethanol at $45^{\circ} \mathrm{C}$ by free and alginateimmobilized preparations of Kluyveromyces marxianus IMB3. Biop. Eng. 18(4): 257-259.

Fresnel, J. \& K. Moore. 1978. Swiss Scientists develop soft drink from whey. Dairy Sci. Abstrs. 41(1):87 
Garibay García, M.; R. Quintero Ramírez y A. Munguia López. 1993. Biotecnología Alimentaria. Limusa Noriega Editores. México, D. F., pp. 152-233.

Gawel, J. \& F. Kosikowski. 1978. Application of acid lactase to wine making from cottage cheese whey concentrate. Dairy Sci. Abst. 41(1):88.

Kriel, J.1980. A chocolate beverage made from sweet whey. Dairy Sci. Abst. $42(6): 3356$.

Marwaha, S; J. Kennedy \& V. Seghal. 1988. Simulation of process conditions of continuous ethanol fermentation of whey permeate using alginate entrapped K. marxianus NCYC 179 cells in a packed-bed reactor system. Process Biochem, pp. 17-22.

Mc Ghee, J.; J. Grant \& R. Detroy. 1982. Continuous and static fermentation of glucose to ethanol by immobilized Saccharomyces cerevisae cells of different ages. Appl. Environ. Microbiol, pp. 19-22.

Molina Ubeda, R. 1992. Técnicas de filtración en la industria enológica. A Madrid Vicente Ediciones, pp. 20-40, 55-64.

NTP 202.083. Jul. 1988. Leche y Productos Lácteos. Ensayos Microbiológicos.

NTP 212.014. Dic 1985. Bebidas alcohólicas. Vinos.

Núñez, M. \& J. Lema. 1987. Cell immobilization: Application to alcohol production. Enzyme Microb. Technol. 9: 642-651.

Ough, C. 1996. Tratado básico de Enología. Acribia S. A. Zaragoza, 294 pp.
Parascandola, P.; E. Alteris; R. Sentandren \& J. Zueco. 1997. Immobilization and ethanol induce the same molecular response at the level of the cell wall in growing yeast. FEMS Microbiology Letters. 150: 121-126.

Pearson, D. 1976. Técnicas de Laboratorio para el Análisis de Alimentos. Acribia S. A. Zaragoza, 331 pp.

Pedrero, D. y R. Pangborn. 1989. Fivaluación sensorial de los alimentos. Alhambra Mexicana S. A. México, D. F, 274 pp.

Peynaud, E. 1989. Enología práctica. Conocimiento y elaboración del vino.Tercera edición. Ediciones Mundi-Prensa. Madrid. pp. 111-137. 291-299, 323-331, 357-367 pp.

Sabaa-Srur, A.; M. Koblitz; L. Freiman; V. Oliveira y E. Gonzales. 1995. L'so integral do soro de queijo minas frescal elaboração de bebida fermentada e sua aceitabilidae. 17 $(l-2): 57-62$

Wiseman, A. 1991. Manual de Biotecnología de las enzimas. Acribia S. A. Zaragoza, pp. 69-76.

Yadav, B.; O. Rani; S. Dhamija; P. Nigam \& D. Singh. 1996. Process optimization for continuous ethanol fermentation by alginate-immobilized cells of Saccharomyces cerevisiae HALI-1. J. Basic Microbiol. 36(3):205-210

Yang, H.; F. Bodyfelt \& K. Berggen. 1979. Utilization of cheese whey for wine production. Dairy Sci. Abst. 41 (4):89.

Yousef, K.; M. Ghareib \& A. Khalil. 1989. Production of etahnol by alginateentrapped Saccharomyces cerevisiae "14-12". Indian J. Exp. Biol 27(2): 121-123. 